\title{
Using kisspeptin to assess GnRH function in an unusual case of primary amenorrhoea
}

\author{
S Vimalesvaran', S Narayanaswamy1, L Yang1, J K Prague1, A Buckley', A D Miras², \\ S Franks², K Meeran² and W S Dhillo'
}

'Department of Endocrinology \& Metabolism, Imperial College London, UK and 2Department of Diabetes \& Endocrinology, Imperial Healthcare NHS Trust, London, UK
Correspondence

should be addressed

to W S Dhillo

Email

w.dhillo@imperial.ac.uk

\section{Summary}

Primary amenorrhoea is defined as the failure to commence menstruation by the age of 15 years, in the presence of normal secondary sexual development. The potential causes of primary amenorrhoea extend from structural to chromosomal abnormalities. Polycystic ovarian syndrome (PCOS) is a common cause of secondary amenorrhoea but an uncommon cause of primary amenorrhoea. An early and prompt diagnosis of PCOS is important, as up to $30 \%$ of these women are predisposed to glucose intolerance and obesity, with the subgroup of women presenting with primary amenorrhoea and PCOS displaying a higher incidence of metabolic dysfunction. We describe a case of an 18-year-old female presenting with primary amenorrhoea of unknown aetiology. Although initial investigations did not demonstrate clinical or biochemical hyperandrogenism or any radiological evidence of polycystic ovaries, a raised luteinising hormone (LH) suggested a diagnosis of PCOS. If PCOS was the correct diagnosis, then one would expect intact hypothalamic GnRH and pituitary gonadotropin release. We used the novel hormone kisspeptin to confirm intact hypothalamic GnRH release and a GnRH stimulation test to confirm intact pituitary gonadotroph function. This case highlights that kisspeptin is a potential unique tool to test $\mathrm{GnRH}$ function in patients presenting with reproductive disorders.

\section{Learning points:}

- Polycystic ovarian syndrome (PCOS) can present with primary amenorrhoea, and therefore, should be considered in the differential diagnosis.

- PCOS is a heterogeneous condition that may present in lean women with few or absent signs of hyperandrogenism.

- GnRH stimulation tests are useful in evaluating pituitary function; however, to date, we do not have a viable test of GnRH function. Kisspeptin has the potential to form a novel diagnostic tool for assessing hypothalamic GnRH function by monitoring gonadotropin response as a surrogate marker of $\mathrm{GnRH}$ release.

- Confirmation of intact GnRH function helps consolidate a diagnosis in primary amenorrhoea and gives an indication of future fertility.

\section{Background}

Primary amenorrhoea is described as the failure to reach menarche. The prevalence of primary amenorrhoea is roughly $3-4 \%(1)$, with highly specialised referral centres reporting only 10-15 patients per annum (2).
Although there are a number of causes of primary amenorrhoea including chromosomal abnormalities or anatomical disorders, there remain a proportion of cases where the cause is difficult to elucidate despite the 
plethora of current endocrine testing. Correct diagnosis of any underlying pathological cause will enable swift treatment at this vulnerable stage of development.

Polycystic ovarian syndrome (PCOS) very commonly presents with secondary amenorrhoea; however, a small proportion present with primary amenorrhoea. PCOS is the commonest endocrine condition in women of reproductive age and affects approximately $6-8 \%$ of this age group (3). It remains a complex and heterogeneous condition. The three main diagnostic classification systems at present are the National Institutes of Health (NIH) criteria, the Rotterdam criteria and the Androgen Excess and PCOS Society Criteria. The classifications concur that the diagnosis of PCOS is based on a combination of three components: hyperandrogenism (clinical or biochemical), chronic anovulation and multiple ovarian follicles on ultrasound $(3,4,5)$.

Over $50 \%$ of patients with PCOS are obese and adult women have a 10 -fold higher likelihood of developing type 2 diabetes and double the risk of metabolic syndrome compared to the normal population (6). Diagnosis in adolescents can be challenging as some features coincide with normal puberty and menarche, including anovulatory cycles, irregular menstrual cycles and features of hyperandrogenism, such as acne (7).

Hamilton et al. described higher risks of developing metabolic syndrome in a subgroup of PCOS patients presenting with primary amenorrhoea compared with those presenting with oligoamenorrhoea (7). This further illustrates the need to accurately recognise patients with primary amenorrhoea and PCOS.

Despite a number of endocrine tests concerning stimulation or suppression of the axis, hypothalamic function testing remains relatively unexplored in common clinical practice. Kisspeptin is a hypothalamic neuropeptide that acts on the kisspeptin receptor expressed on GnRH neurons to stimulate GnRH release
(8). In 2003, mutations of the kisspeptin receptor found in 2 separate consanguineous families were identified in whom mutations of the kisspeptin receptor resulted in affected members presenting with hypogonadotrophic hypogonadism $(9,10)$, showing for the first time the importance of kisspeptin upstream of GnRH signalling. Since then, an explosion of kisspeptin research has dominated the field of reproductive neuroendocrinology. Kisspeptin administration peripherally has been shown to act at the median eminence (devoid of blood-brain barrier) to stimulate GnRH neurons to secrete GnRH and subsequently gonadotropins (11). We have administered kisspeptin to healthy men and women as well as to those with hypothalamic amenorrhoea and have shown an increase in gonadotropins (12). Kisspeptin might therefore be used in cases of primary amenorrhoea as a novel test of hypothalamic GnRH function.

\section{Case presentation}

An 18-year-old Caucasian female presented with primary amenorrhoea, but no other significant symptoms. Pubertal development had been entirely normal with normal developmental milestones and no past medical history. She started wearing bras at age 11 years and developed secondary sexual hair at approximately the same time. The patient's mother and sister both went through menarche at age 13 years, and there was no other significant family history.

Examination revealed normal BMI of 22.6 with her weight remaining steady for some time. She displayed normal secondary sexual characteristics (Tanner Stage 5) and there were no phenotypic features of polycystic ovarian syndrome, congenital adrenal hyperplasia or Turner's syndrome. Specifically, she did not show signs of virilisation (such as hirsuitism), increased muscle mass or sexual ambiguity.

Table 1 Results of hormone profile.

\begin{tabular}{|c|c|c|}
\hline & 03/13 & $09 / 13$ \\
\hline LH (IU/mL) & 14.1 & 14.6 \\
\hline FSH (IU/mL) & 5.0 & 4.5 \\
\hline LH/FSH ratio & 2.82 & 3.24 \\
\hline Oestradiol (pmol/L) & 114 & 92 \\
\hline \multicolumn{3}{|l|}{ Progesterone (nmol/L) } \\
\hline \multicolumn{3}{|c|}{ Anti-Mullerian hormone (pmol/L) } \\
\hline Testosterone (nmol/L) & 2.6 & \\
\hline SHBG (nmol/L) & 34 & \\
\hline Free androgen index & 7.6 & \\
\hline Prolactin (U/L) & 314 & \\
\hline
\end{tabular}

\begin{tabular}{l}
\hline $\mathbf{1 1 / 1 3}$ \\
\hline 4.1 \\
3.9 \\
68.1 \\
\hline
\end{tabular}

\begin{tabular}{l}
\hline $\mathbf{0 3 / 1 4}$ \\
\hline 16.4 \\
5.2 \\
3.15 \\
122 \\
68.0
\end{tabular}

\begin{tabular}{l}
\hline $\mathbf{1 2 / 1 4}$ \\
\hline 11.1 \\
1.9 \\
5.84 \\
343
\end{tabular}

\begin{tabular}{l}
\hline $\mathbf{1 2 / 1 5}$ \\
\hline 15.7 \\
4.9 \\
3.20 \\
124 \\
1 \\
1.2 \\
28 \\
4.2 \\
149 \\
\hline
\end{tabular}

Reference $2-10$ $1.5-10$ $0-300$ $0-5$ $10-40$ $0-2$ 30-100 $7-10$ $100-500$ 


\section{Investigation}

Her basal endocrine blood test results are shown in Table 1 . A pregnancy test was negative. Karyotype testing revealed a normal female 46XX karyotype. A pelvic ultrasound showed normal uterine and ovarian architecture; with a normal endometrial thickness.

She had a DEXA scan which showed a lumbar $Z$-score -1.0 and a left wrist $\mathrm{X}$-ray which showed fused bones consistent with a normal adult wrist.

She received a 10-day course of medroxyprogesterone acetate from which she had a withdrawal bleed, indicating normal uterine function.

Her basal blood tests showed a raised LH, inverse LH:FSH ratio and a borderline raised testosterone on only one occasion suggestive of PCOS. However, phenotypically she was lean with no features of hyperandrogenism. If PCOS was the correct diagnosis then one would expect intact hypothalamic GnRH and pituitary gonadotropin release.

To assess her hypothalamic GnRH function, she underwent an 8-h assessment of gonadotropins beginning at 09:00 $\mathrm{h}$ with blood sampling at 10-min intervals until 17:00 $\mathrm{h}$. This is the gold standard test for assessment of LH pulsatility. As shown in Fig. 1, she has a normal pattern of LH pulsatility; however, the amplitude of LH pulses and baseline LH was elevated (normal LH pulse frequency $7.0 \pm 1.8$ pulse $/ 12 \mathrm{~h} ;$ amplitude $2.3 \pm 1.0 \mathrm{IU} / \mathrm{mL}$; normal range for LH 2-10IU/mL) (13). She returned for another 8-h study during which kisspeptin-54 was administered via intravenous infusion in escalating doses every $2 \mathrm{~h}$ (Fig. 2). She showed a normal response to kisspeptin-54 with increasing LH pulsatility as well as an elevation in the LH baseline, indicating normal GnRH secretion in response to kisspeptin-54. She had a normal response

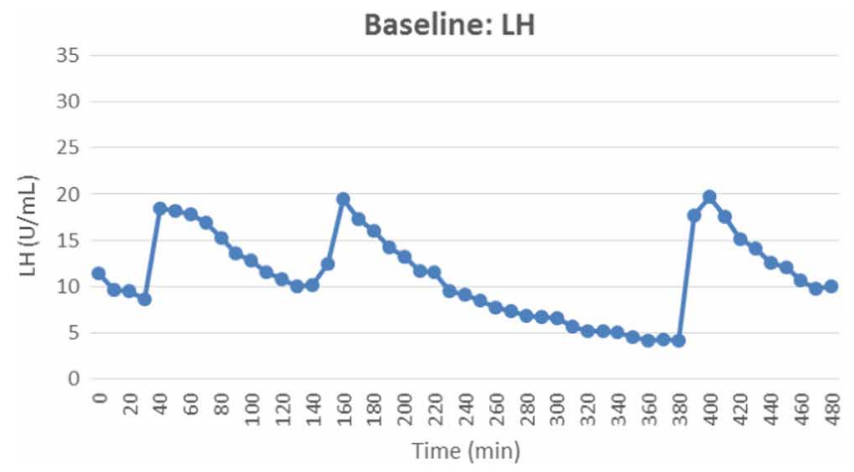

Figure 1

Baseline LH profile over $8 \mathrm{~h}$. Blood samples collected for $\mathrm{LH}$ analysis at 10-min intervals from 09:00 $\mathrm{h}$ for $8 \mathrm{~h}$ to determine LH pulsatility.

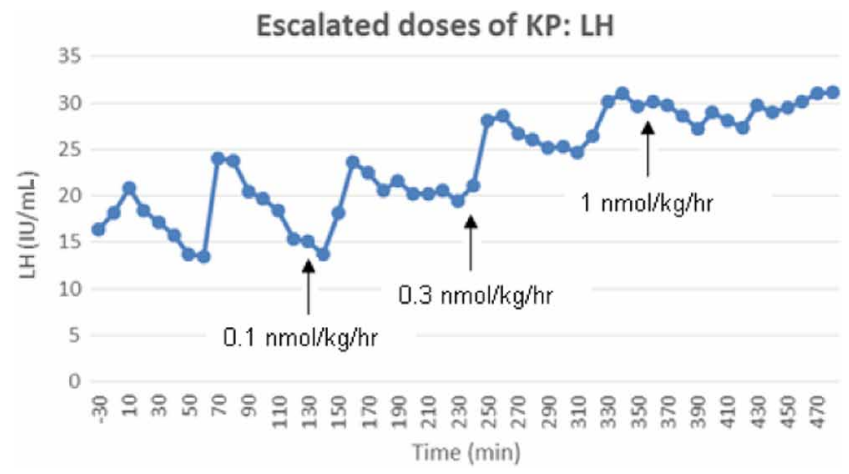

Figure 2

$\mathrm{LH}$ response to escalating doses of kisspeptin- 54 administered by intravenous infusion over $8 \mathrm{~h}$.

to a GnRH stimulation test (Table 2), indicating normal pituitary gonadotroph function.

\section{Treatment}

She has been started on oral contraceptive medication (Microgynon) to provide oestrogen and progesterone replacement as a means of achieving regular monthly withdrawal bleeds.

\section{Outcome and follow-up}

She is receiving regular on-going review in clinic with the Endocrine team.

\section{Discussion}

Adolescents with PCOS presenting with primary amenorrhoea are a distinct subgroup, with higher prevalence of biochemical hyperandrogenism and a higher risk of dyslipidaemia despite body habitus, compared to PCOS patients with oligomenorrhoea. There is also evidence of increased insulin resistance and higher BMI-adjusted fasting triglyceride concentrations in these individuals (7). Hence, an early and prompt diagnosis of PCOS is important.

In this case, the patient did not demonstrate any clinical or significant biochemical features of hyperandrogenism.

Table 2 Results of gonadotropin-releasing hormone stimulation test.

\begin{tabular}{|c|c|c|c|c|}
\hline & Baseline & $30 \mathrm{~min}$ & $60 \mathrm{~min}$ & Reference \\
\hline LH (IU/L) & 14.6 & 58.3 & 45.3 & $>10$ \\
\hline FSH (IU/L) & 4.5 & 9.0 & 8.8 & $>2$ \\
\hline
\end{tabular}


This further illustrates the heterogeneous presentation of PCOS and the variability in potential presenting features.

We used the novel hormone kisspeptin to confirm intact hypothalamic GnRH function in our patient. Kisspeptin is a 54-amino acid peptide encoded by the KISS1 gene in humans and acts via the G-proteincoupled receptor (GPR) GPR54 (8). Kisspeptin stimulates GnRH neurons to secrete GnRH leading to the release of gonadotropins and mutations in the gene KISS1R result in pubertal failure $(9,10)$. LH pulsatility in our patient was normal $(7.0 \pm 1.8$ pulses $/ 12 \mathrm{~h})$ although the baseline LH was raised (13). She also responded normally to intravenous kisspeptin with an increase in LH pulsatility and a rise in the baseline, showing that she has intact hypothalamic GnRH function. The GnRH test confirmed normal pituitary function.

Our patient went through puberty at the correct time indicating that her kisspeptin neurons are likely to be intact as kisspeptin is integral to the pubertal process (10). She had a normal karyotype and pelvic ultrasound, ruling out common genetic and structural abnormalities. With dynamic tests confirming normal hypothalamic and pituitary function but a raised $\mathrm{LH}$, a diagnosis of PCOS presenting with primary amenorrhoea was most likely.

This case amplifies the need to consider PCOS as a differential diagnosis for patients presenting with primary amenorrhoea. In an atypical case such as this, where a particular phenotype is not apparent, it is important to exclude other potential causes with investigations such as karyotyping, hormonal testing and endocrine stimulation testing. Disorders of GnRH function or reserve, namely, congenital hypogonadotrophic hypogonadism, have been difficult to investigate in clinical settings as we currently do not have a reliable test for hypothalamic GnRH function. Although it is not yet available in clinical practice, in this case, we demonstrate the use of kisspeptin as a novel test of GnRH activity, with a normal LH response excluding defects in the hypothalamic GnRH centre. In future, it is hoped that kisspeptin will be the test of choice for ruling out abnormal GnRH function in selected cases of primary amenorrhoea with unclear cause.

\section{Patient's perspective}

We welcome comments from your patient; their own description of their experience may help other patients or clinicians who are dealing with a similar problem. If your patient would like to contribute please ensure they include only relevant personal details. Patients may describe their symptoms, how any tests and treatments affected them and how the problem is now.
Declaration of interest

The authors declare that there is no conflict of interest that could be perceived as prejudicing the impartiality of the research reported.

\section{Funding}

Prof. Waljit Dhillo is supported by an NIHR Research professorship (RP-2014-05-001, 2015-2020).

\section{Patient consent}

Informed, written consent has been obtained from the patient, and a scanned copy of the signed consent form is included with this submission.

\section{Author contribution statement}

S Vimalesvaran, S Narayanaswamy and L Yang researched and composed the manuscript. J K Prague and A Buckley were involved with LH pulsatility and kisspeptin infusion studies, data collection and analysis. A D Miras, S Franks, K Meeran and W S Dhillo were involved in the clinical assessment, investigation and on-going management of the patient. All authors contributed towards the finished manuscript including tables and figures.

\section{References}

1 Herman-Giddens ME, Slora EJ, Wasserman RC, Bourdony CJ \& Bhapkar MV 1997 Secondary sexual characteristics and menses in young girls seen in office practice: a study from the Pediatric Research in Office Settings network. Paediatrics 99 505-512. (doi:10.1542/ peds.99.4.505)

2 Pettersson F \& Fries H 1973 Epidemiology of secondary amenorrhoea. Incidence and prevalence rates. American Journal of Obstetrics and Gynecology 117 80-86. (doi:10.1016/0002-9378(73)90732-1)

3 Azziz R, Carmina E, Dewailly D, Diamanti-Kandarakis E, EscobarMorreale F, Futterweit W, Janssen O, Legro R, Norman R, Taylor A, et al. 2009 The androgen excess and PCOS society criteria for the polycystic ovary syndrome: the complete task force report. Fertility and Sterility 91 456-488. (doi:10.1016/j.fertnstert.2008.06.035)

4 Rotterdam ESHRE/ASRM-Sponsored PCOS Consensus Workshop Group 2004 Revised 2003 consensus on diagnostic criteria and long-term health risks related to polycystic ovary syndrome (PCOS). Human Reproduction 19 41-47. (doi:10.1093/humrep/deh098)

5 Zawadski JK \& Dunaif A 1992 Diagnostic criteria for polycystic ovary syndrome: towards a rational approach. In Polycystic Ovary Syndrome, pp. 377-384. Eds A Dunaif, JR Givens \& F Haseltine. Boston, USA: Blackwell Scientific.

6 Bekx M, Connor E \& Allen D 2010 Characteristics of adolescents presenting to a multidisciplinary clinic for polycystic ovarian syndrome. Journal of Pediatric and Adolescent Gynecology 23 7-10. (doi:10.1016/j.jpag.2009.04.004)

7 Rachmiel M, Kives S, Atenafu E \& Hamilton J 2008 Primary amenorrhea as a manifestation of polycystic ovarian syndrome in adolescents: a unique subgroup? Archives of Pediatrics and Adolescent Medicine 162 521-525. (doi:10.1001/archpedi.162.6.521)

8 Kotani M, Detheux M, Vandenbogaerde A, Communi D, Vanderwinden JM, Le Poul E, Brézillon S, Tyldesley R, Suarez-Huerta N, Vandeput F, et al. 2001 The metastasis suppressor gene KiSS-1 encodes kisspeptins, the natural ligands of the orphan G protein-coupled receptor GPR54. Journal of Biological Chemistry $\mathbf{2 7 6}$ 34631-34636. (doi:10.1074/jbc.M104847200) 
9 de Roux N, Genin E, Carel JC, Matsuda F, Chaussain JL \& Milgrom E 2003 Hypogonadotropic hypogonadism due to loss of function of the KiSS1-derived peptide receptor GPR54. PNAS 100 10972-10976. (doi:10.1073/pnas.1834399100)

10 Seminara SB, Messager S, Chatzidaki EE, Thresher RR, Acierno JS, Shagoury JK, Bo-Abbas Y, Kuohung W, Schwinof KM, Hendrick AG, et al. 2003 The GPR54 gene as a regulator of puberty. New England Journal of Medicine 349 1614-1627. (doi:10.1056/NEJMoa035322)

11 d'Anglemont de Tassigny X, Fagg LA, Carlton MB \& Colledge WH 2008 Kisspeptin can stimulate gonadotropin-releasing hormone
$(\mathrm{GnRH})$ release by a direct action at $\mathrm{GnRH}$ nerve terminals. Endocrinology 149 3926-3932. (doi:10.1210/en.2007-1487)

12 Yang L \& Dhillo W 2016 Kisspeptin as a therapeutic target in reproduction. Expert Opinion on Therapeutic Targets 20 567-575. (doi:10.1517/14728222.2016.1124858)

13 Shaw ND, Seminara SB, Welt CK, Au MG, Plummer L, Hughes VA, Dwyer AA, Martin KA, Quinton R, Mericq V, et al. 2011 Expanding the phenotype and genotype of female GnRH deficiency. Journal of Clinical Endocrinology and Metabolism 96 E566-E576. (doi:10.1210/ jc.2010-2292)

Received in final form 23 January 2017 Accepted 10 February 2017 